\title{
Multiuser Linear Precoding for Cooperating Base Stations with Asynchronous Interference
}

\author{
Imad H. Azzam \\ Dept. of Elec. and Comp. Eng. \\ University of Toronto \\ 10 King's College Road, \\ Toronto, ON, Canada M5S 3G4. \\ Email: iazzamecomm. utoronto.ca
}

\author{
Raviraj S. Adve \\ Dept. of Elec. and Comp. Eng. \\ University of Toronto \\ 10 King's College Road, \\ Toronto, ON, Canada M5S 3G4. \\ Email: rsadvedcomm. utoronto.ca
}

\begin{abstract}
This paper addresses multiuser linear precoding using multiple cooperating base stations (BSs). All BSs, and potentially the users, are equipped with multiple antennas. Prior work in multiuser precoding with a single BS extensively uses a downlink/uplink duality that significantly reduces the computation load. Here we show that the presence of asynchronous interference, unfortunately always present due to multiple BSs, precludes a simple duality. This in turn complicates deriving the needed precoding matrices. Even when duality is assumed to exist, asynchronous interference has its implications on the convexity of the power allocation problem. An optimization formulation and several related simulations for the asynchronous case are presented, including one where duality is assumed and another wherein orthogonal frequency division multiplexing (OFDM) is used for transmission and its cyclic prefix is used to mitigate asynchronous reception for edge users.
\end{abstract}

\section{INTRODUCTION}

Future wireless networks will demand better performance than today's networks, aiming at higher data rates, lower error rates, and a more efficient and flexible use of limited bandwidth and power resources. It is now well accepted that multiple input, multiple output, (MIMO) systems will be required to meet these goals. Maximizing flexibility will, in turn, require multiuser communications wherein a single base station (BS) transmitter communicates with multiple users simultaneously, i.e., the multiuser downlink.

When channel state information (CSI) is available at the transmitter, MIMO system performance can be further improved by pre-distorting the transmission to best match the available CSI. This paper focuses on linear precoding [1][5] where the signals to be transmitted are multiplied with a precoding matrix before transmission. Similarly, the receiver multiplies the received signal with a decoding matrix to minimize multiuser interference. While the early works focused on minimizing the sum of the mean squared error (SMSE) across all users' signals [1]-[4], linear precoding to maximize sum data rate is also possible [5].

Most of the work in precoding is based on a duality between the multiuser downlink and a virtual multiuser uplink [2][4]. The duality states that, given a power allocation at the transmitter (in the downlink and uplink), the same sum mean squared errors (SMSE) can be achieved using the same precoding/decoding matrices. This duality was shown for the single receive antenna case in [2] and generalized to the MIMO scenario in [4]. This downlink/uplink duality suggests the precoding and decoding matrices can be obtained via receive processing only, i.e., using the Weiner filter [4]. The outstanding issue is then power allocation across users, which is a convex optimization problem when minimizing SMSE [4].

More recently researchers have begun investigating the notion of multiple BSs cooperating to achieve more efficient use of bandwidth. The ultimate goal of such systems is a frequency reuse factor of unity. In this multi-BS, multiuser precoding scenario, multiple BSs coordinate their transmissions to a group of users (that may straddle a traditional cell boundary). This cooperation can provide better system performance, especially when servicing cell-edge users. The work in [6] is probably the first to discuss multiuser communications with multiple BSs. Recent work has investigated linear precoding for multiple cooperating BSs [7], the subject of this paper.

When dealing with multi-cell environments, a crucial assumption often made is that both the desired and interfering signals arrive synchronously at each user [7]-[10]. However, synchronous interference is physically impossible [11]. The work in [11] provides amendments to some existing algorithms accounting for this asynchronous interference. In this paper we show that this asynchronous interference significantly undermines the downlink/uplink duality on which most linear precoding theory is based. Furthermore, the convexity of the power allocation problem is unclear, i.e., linear precoding for the multiuser, multiple cooperating BS case is significantly more complex than has been previously supposed.

In this paper, building on the work in [4], we formulate an algorithm that minimizes the SMSE under sum power constraints. Throughout, the analysis accounts for the asynchronous interference introduced by using multiple BSs. The contributions of this paper are:

- Presenting an uplink system model that accounts for the asynchronous interference introduced by the presence of cooperating BSs.

- Illustrating that, in the case with multiple BSs and asynchronous interference, a simple downlink/uplink duality does not exist and that the power allocation problem is 
not provably convex. It is only simulations that suggest that convexity holds.

- Formulating the optimization problem for the case of asynchronous interference, presenting the achievable performance in this scenario, and using simulations to illustrate the performance loss by assuming synchronous interference.

This paper is organized as follows: In Section II the uplink and downlink system models are described. In Section III, downlink/uplink duality is discussed as relating to the case of cooperating base stations and related issues are developed. In Section IV, the results of the simulations are presented. Finally, the paper concludes with some remarks in Section V.

\section{System MODEL}

This section describes the system models of the multiuser downlink and the uplink. In both cases it is assumed that there are $B$ BSs and $K$ users randomly distributed in the cells of the $B$ BSs. Each BS has $M$ antennas, while user $k$ has $N_{k}$ antennas with $N=\sum_{k=1}^{K} N_{k}$. Moreover, each user transmits or receives $L_{k}$ data streams simultaneously, where $L_{k} \leq \min \left\{M, N_{k}\right\}$. Also, $L=\sum_{k=1}^{K} L_{k}$. Note that all data streams share the same frequency and time channels. The BSs communicate to the users over frequency flat channels. All BSs are assumed to know the CSI to all users perfectly.

\section{A. Downlink System Model}

Let $\mathbf{x}_{k}\left(L_{k} \times 1\right)$ be the column data vector containing user $k$ 's data streams to be transmitted from all BSs. It is assumed that the data vectors of different users are independent, i.e., $\mathbf{E}\left[\mathbf{x}_{k} \mathbf{x}_{j}^{H}\right]=\mathbf{0}, \forall j \neq k$, where $(\cdot)^{H}$ is the Hermitian operator and $\mathbf{E}[\cdot]$ denotes the expectation operator. Also the data symbols in $\mathbf{x}_{k}$ are assumed independent with unit average power, i.e., $\mathbf{E}\left[\mathbf{x}_{k} \mathbf{x}_{k}^{H}\right]=\mathbf{I}_{L_{k}}$. Before transmission, $\mathbf{x}_{k}$ is allocated a certain power by multiplying it by $\sqrt{\mathbf{P}_{k}}\left(L_{k} \times L_{k}\right)$, where $\mathbf{P}_{k}$ is a diagonal matrix, whose components are the powers allocated to the different components of $\mathbf{x}_{k}$. Furthermore, before transmission from BS $b$, the data vector meant for user $k$ is linearly precoded with a matrix $\mathbf{U}_{k}^{(b)}\left(M \times L_{k}\right)$. The channel between BS $b$ and user $k$ is represented by the matrix $\mathbf{H}_{k}^{(b)}$ $\left(M \times N_{k}\right)$, whose elements are circularly symmetric complex Gaussian random variables with unit variance. These channels are assumed known at the $B$ BSs. The $N_{k} \times 1$ signal received by user $k$ is

$$
\mathbf{y}_{k}=\sum_{b=1}^{B} \mathbf{H}_{k}^{(b)^{H}} \mathbf{U}_{k}^{(b)} \sqrt{\mathbf{P}_{k}} \mathbf{x}_{k}+\text { interference }+\mathbf{n}_{k},
$$

where $\mathbf{n}_{k}$ denotes the additive white Gaussian noise (AWGN). The interference term is explained in more detail below. Finally, user $k$ processes its received signal linearly by multiplying it by a decoding matrix $\mathbf{V}_{k}^{H}\left(L_{k} \times N_{k}\right)$ to produce $\hat{\mathbf{x}}_{k}=$ $\mathbf{V}_{k}^{H} \mathbf{y}_{k}$. The MSE for user $k$ is defined as $\mathbf{E}\left[\left\|\mathbf{x}_{k}-\hat{\mathbf{x}}_{k}\right\|^{2}\right]$.

The interference term in (1) is described in some detail below. As for the first summation in (1), it is assumed that the transmissions from all BSs meant for a certain user $k$
$\left(\mathbf{t}_{k}^{(b)}=\mathbf{U}_{k}^{(b)} \sqrt{\mathbf{P}_{k}} \mathbf{x}_{k}\right.$, for $\left.b=1, \ldots, B\right)$ are received by user $k$ synchronously. Therefore, as in [11], BS $b$ advances the time at which it transmits $\mathbf{t}_{k}^{(b)}$ by $\tau_{k}^{(b)}-\tau_{k}^{\left(b_{k}\right)}$, where $\tau_{k}^{(b)}$ is the propagation delay from BS $b$ to user $k$, and $\tau_{k}^{\left(b_{k}\right)}$ is the propagation delay from user $k$ to the nearest BS. Due to the random distribution of users, each user will be at a different distance from the different BSs. Since each user receives its own data from the different BSs synchronously, it is physically impossible that the interference at user $k$ from other users in the system be synchronous with user $k$ 's intended signals as well; the interference is therefore asynchronous. This causes the pulse shape used to transmit interfering data streams to be misaligned with the matched filter at user $k$. Adapting from [11], this misalignment can expressed as:

$$
\text { interference }=\sum_{b=1}^{B} \mathbf{H}_{k}^{(b)^{H}} \sum_{\substack{j=1 \\ j \neq k}}^{K} \mathbf{U}_{j}^{(b)} \sqrt{\mathbf{P}_{j}} \mathbf{i}_{j k}^{(b)},
$$

where

$$
\begin{gathered}
\mathbf{i}_{j k}^{(b)}(m)=\rho\left(\delta_{j k}^{(b)}-T_{S}\right) \mathbf{x}_{j}\left(m_{j k}^{(b)}\right)+\rho\left(\delta_{j k}^{(b)}\right) \mathbf{x}_{j}\left(m_{j k}^{(b)}+1\right), \\
\rho(\tau)=\int_{0}^{T_{S}} g(t) g(t-\tau) d t, \quad \delta_{j k}^{(b)}=\tau_{j k}^{(b)} \bmod T_{S} \\
\tau_{j k}^{(b)}=\tau_{k}^{(b)}-\tau_{k}^{\left(b_{k}\right)}-\left(\tau_{j}^{(b)}-\tau_{j}^{\left(b_{j}\right)}\right)
\end{gathered}
$$

Here, $g(t)$ is the pulse shaping filter, and $T_{S}$ is the symbol period. The term $\mathbf{i}_{j k}^{(b)}$ is the misaligned interference caused on user $k$ when BS $b$ transmits data streams to user $j$. The term $\tau_{j k}^{(b)}$ represents the difference between the time when user $k$ receives its intended signal and the time when interference from BS $b$ while transmitting to user $j$ arrives at user $k$. Its expression can be understood as follows. Assume all BSs transmit to their closest users at the same time $(t=0)$. User $k$ receives its intended signal at $t=\tau_{k}^{\left(b_{k}\right)}$. The propagation delay from BS $b$ to user $k$ is $\tau_{k}^{(b)}$ and hence $\tau_{j k}^{(b)}$ should be $\tau_{k}^{(b)}-\tau_{k}^{\left(b_{k}\right)}$; but BS $b$ is transmitting to user $j$ and thus has advanced its transmission by $\tau_{j}^{(b)}-\tau_{j}^{\left(b_{j}\right)}$ so that user $j$ receives all its intended signals from all BSs simultaneously. Therefore, the interference arrives $\tau_{j}^{(b)}-\tau_{j}^{\left(b_{j}\right)}$ seconds earlier, making $\tau_{j k}^{(b)}=\tau_{k}^{(b)}-\tau_{k}^{\left(b_{k}\right)}-\left(\tau_{j}^{(b)}-\tau_{j}^{\left(b_{j}\right)}\right)$. Therefore,

$$
\begin{aligned}
\hat{\mathbf{x}}_{k}= & \mathbf{V}_{k}^{H} \sum_{b=1}^{B} \mathbf{H}_{k}^{(b)^{H}} \mathbf{U}_{k}^{(b)} \sqrt{\mathbf{P}_{k}} \mathbf{x}_{k} \\
& +\mathbf{V}_{k}^{H} \sum_{b=1}^{B} \mathbf{H}_{k}^{(b)^{H}} \sum_{\substack{j=1 \\
j \neq k}}^{K} \mathbf{U}_{j}^{(b)} \sqrt{\mathbf{P}_{j}} \mathbf{i}_{j k}^{(b)}+\mathbf{V}_{k}^{H} \mathbf{n}_{k}
\end{aligned}
$$

\section{B. Virtual Uplink System Model}

As mentioned previously, many works make use of an downlink/uplink duality because of the simplification it provides in obtaining $\mathbf{U}_{k}$ and $\mathbf{V}_{k}$. Since we hope to exploit this duality for our case, we present in this section the virtual uplink system model to be used. 
In the uplink, the system model is very similar to that in the downlink, except for the following changes: $\mathbf{y}_{k}^{(b)}(M \times 1)$ is the received signal at $\mathrm{BS} b$, and $\mathbf{y}_{k}=\left[\mathbf{y}_{k}^{(1)^{T}} \ldots \mathbf{y}_{k}^{(B)^{T}}\right]^{T}$ $(B M \times 1)$ is a vertical concatenation of the the received signals at all BSs. $\mathbf{V}_{k}$ is now the precoding matrix used by user $k$, and $\mathbf{U}_{k}^{(b)}$ is the decoding matrix used by BS $b$ to process its received signal from user $k$. The power allocation matrix used by user $k$ is $\mathbf{Q}_{k}$. As for timing issues, the different BSs receive user $k$ 's transmission at different times. It is assumed that a BS can buffer its received signal and delay the processing until all other BSs have received user $k$ 's transmission, after which joint decoding is done. In this case, the interference term $\mathbf{i}_{j k}^{(b)}(m)$ changes to $\mathbf{e}_{j k}^{(b)}(m)$, which has the exact same form as $\mathbf{i}_{j k}^{(b)}(m)$, but $\tau_{j k}^{(b)}=\tau_{j}^{(b)}-\tau_{k}^{(b)}$. Overall, $\hat{\mathbf{x}}_{k}$ can be expressed as follows.

$$
\begin{aligned}
\hat{\mathbf{x}}_{k} & =\sum_{b=1}^{B} \mathbf{U}_{k}^{(b)^{H}} \mathbf{H}_{k}^{(b)} \mathbf{V}_{k} \sqrt{\mathbf{Q}_{k}} \mathbf{x}_{k} \\
& +\sum_{\substack{j=1 \\
j \neq k}}^{K} \mathbf{U}_{k}^{(b)^{H}} \mathbf{H}_{j}^{(b)} \mathbf{V}_{j} \sqrt{\mathbf{Q}_{j}} \mathbf{e}_{j k}^{(b)}+\sum_{b=1}^{B} \mathbf{U}_{k}^{(b)^{H}} \mathbf{n}^{(b)}
\end{aligned}
$$

The term $\mathbf{e}_{j k}^{(b)}$ is the misaligned interference caused by user $j$ 's signal on user $k$ 's signal when both are being received by BS $b$. The term $\tau_{j k}^{(b)}$ represents the difference between the time when BS $b$ starts receiving user $j$ 's signal and the time when it starts receiving user $k$ 's signal. In order to understand the expression of $\tau_{j k}^{(b)}$ in the uplink, assume that all the users transmit their data streams at the same time, call it $t=0$ (just like BSs transmitted to their closest users at the same time in the downlink). The interfering signal from user $j$ arrives at BS $b$ at $t=\tau_{j}^{(b)}$, while the intended signal from user $k$ arrives at $t=\tau_{k}^{(b)}$. Therefore, the difference is $\tau_{j k}^{(b)}=\tau_{j}^{(b)}-\tau_{k}^{(b)}$.

\section{DOWNLINK/UPLINK DUALITY}

The existence of a downlink/uplink duality can help simplify algorithmic solutions for a given problem. In the case at hand, the duality states that the same MSEs can be obtained in the uplink and downlink with the same power constraint [2][4]. In this section we show that the duality, so useful with synchronous communications, is not available in our case of cooperating BSs. In general, proving the existence of a downlink/uplink duality requires two steps. First, signal-tointerference-plus-noise ratio (SINR) targets are set on each data stream and the proof shows that same total power is needed to meet these targets in the downlink and uplink. In the second step, the proof requires showing that the MSE for any data stream is the same, whether in the downlink or uplink, using the fact that the uplink and downlink SINR are the same.

For the first step, let the target SINR for data stream $j$ of user $k$ be $\Gamma_{k j}$. We would like to find a power allocation vector p across all data streams such that the minimum of the ratios $\operatorname{SINR}_{k j}^{D L} / \Gamma_{k j}$ over all values of $k$ and $j$ is maximized and $\|\mathbf{p}\|_{1}=\mathbf{1}^{T} \mathbf{p} \leq P_{\max }$, where $\mathbf{1}$ is an all-ones $L \times 1$ vector. It was shown in [2] that the solution of this problem makes all the above ratios equal to the same level, denoted as $C^{D L}$ :

$$
C^{D L}=\frac{\operatorname{SINR}_{k j}^{D L}}{\Gamma_{k j}}, \quad 1 \leq k \leq K, 1 \leq j \leq L_{k},\|\mathbf{p}\|_{1} \leq P_{\text {max }}
$$

From (8), we can write the following equation for any data stream $j$ of user $k$.

$$
p_{k j} \frac{1}{C^{D L}}=p_{k j} \frac{\Gamma_{k j}}{\operatorname{SINR}_{k j}^{D L}}
$$

Following from the system model, the SINR of a data stream $j$ of user $k$ in the downlink can be expressed as follows,

$$
\operatorname{SINR}_{k j}^{D L}=p_{k j} \frac{\mathbf{v}_{k j}^{H} \mathbf{S}_{k j}^{D L} \mathbf{v}_{k j}}{\mathbf{v}_{k j}^{H} \mathbf{T}_{k j}^{D L} \mathbf{v}_{k j}}
$$

where

$$
\mathbf{S}_{k j}^{D L}=\left(\sum_{b=1}^{B} \mathbf{H}_{k}^{(b)^{H}} \mathbf{u}_{k j}^{(b)}\right)\left(\sum_{b=1}^{B} \mathbf{u}_{k j}^{(b)^{H}} \mathbf{H}_{k}^{(b)}\right),
$$

where $\mathbf{u}_{k j}^{(b)}$ denotes the column of $\mathbf{U}_{k}$ corresponding to the $j$-th stream of the $k$-th user at the BS $b$. Also,

$$
\begin{aligned}
\mathbf{T}_{k j}^{D L} & =\sum_{\substack{l=1 \\
l \neq j}}^{L_{k}} p_{k l}\left(\sum_{b=1}^{B} \mathbf{H}_{k}^{(b)^{H}} \mathbf{u}_{k l}^{(b)}\right)\left(\sum_{b=1}^{B} \mathbf{u}_{k l}^{(b)^{H}} \mathbf{H}_{k}^{(b)}\right)+\sigma^{2} \mathbf{I}_{N_{k}} \\
+ & \mathbf{E}\left[\sum_{\substack{c=1 \\
c \neq k}}^{K} \sum_{l=1}^{L_{c}} p_{c l}\left(\sum_{b=1}^{B} \mathbf{H}_{k}^{(b)^{H}} \mathbf{u}_{c l}^{(b)} \mathbf{i}_{c k j}^{(b)}\right)\left(\sum_{b=1}^{B} \mathbf{i}_{c k j}^{(b)^{H}} \mathbf{u}_{c l}^{(b)^{H}} \mathbf{H}_{k}^{(b)}\right)\right] .
\end{aligned}
$$

The third subscript of $\mathbf{i}_{c k j}^{(b)}$ is the index of a certain data stream inside $\mathbf{i}_{c k}^{(b)}$. Note that it is assumed, as in [7], that the covariance matrix of any external interference can be estimated, for example by training, and whitened. The whitening filter can be considered as part of global channel matrix of user $k$, $\mathbf{H}_{k}=\left[\mathbf{H}_{k}^{(1)^{T}} \mathbf{H}_{k}^{(2)^{T}} \ldots \mathbf{H}_{k}^{(B)^{T}}\right]^{T}$. Hence, we only include a scaled identity matrix in (12) to represent noise and external interference. Next, the $L$ equalities given by (9) can be grouped together in one equation as follows

$$
\mathbf{p} \frac{1}{C^{D L}}=\mathbf{D} \Psi^{D L} \mathbf{p}+\mathbf{D} \sigma^{D L}
$$

where

$$
\begin{gathered}
\mathbf{D}=\operatorname{diag}\left(\frac{\Gamma_{11}}{\mathbf{v}_{11}^{H} \mathbf{S}_{11}^{D L} \mathbf{v}_{11}}, \ldots, \frac{\Gamma_{K L_{K}}}{\mathbf{v}_{K L_{k}}^{H} \mathbf{S}_{K L_{K}}^{D L} \mathbf{v}_{K L_{K}}}\right) \\
{\left[\Psi^{D L}\right]_{L_{\text {row }} L_{\text {col }}}=} \\
\mid \begin{array}{l}
\left.\mathbf{v}_{k_{\text {row }} l_{\text {row }}}^{H} \mathbf{E}\left[\sum_{b=1}^{B} \mathbf{H}_{k_{\text {row }}}^{(b)^{H}} \mathbf{u}_{k_{\text {col }} l_{\text {col }}}^{(b)} \mathbf{i}_{k_{\text {col }} k_{\text {row }} l_{\text {row }}}^{(b)}\right]\right|^{2} \\
1 \leq L_{\text {row }}, L_{\text {col }} \leq L \text { and } L_{\text {row }} \neq L_{\text {col }}
\end{array} \\
\sigma^{D L}=\sigma^{2}\left[\mathbf{v}_{11}^{H} \mathbf{v}_{11}, \ldots, \mathbf{v}_{K L_{K}}^{H} \mathbf{v}_{K L_{K}}\right]^{T}=\sigma^{2} \mathbf{1} .
\end{gathered}
$$


Here, $k_{\text {row }}$ is the user to which data stream $L_{\text {row }}$ belongs when the data streams are labeled from 1 to $L ; l_{\text {row }}$ is the index of that data stream relative to user $k_{\text {row }}$. The same applies to $k_{c o l}$ and $l_{c o l}$. Note that the diagonal entries of $\left[\Psi^{D L}\right]$ are zero. Also, it is assumed, for ease of representation, that $\mathbf{i}_{k k}^{(b)}=\mathbf{x}_{k}$, and in that case $\left[\Psi^{D L}\right]_{L_{\text {row }} L_{\text {col }}}$ simplifies to the following expression,

$$
\left[\Psi^{D L}\right]_{L_{\text {row }} L_{\text {col }}}=\left|\mathbf{v}_{k_{\text {row }} l_{\text {row }}}^{H} \sum_{b=1}^{B} \mathbf{H}_{k_{\text {row }}}^{(b)^{H}} \mathbf{u}_{k_{\text {col }} l_{\text {col }}}^{(b)}\right|^{2} .
$$

To minimize the total power while meeting the target SINRs at the same time, $C^{D L}$ is set to 1 . Accordingly, and after simple mathematical manipulations to (13), we get

$$
\mathbf{p}=\sigma^{2}\left(\mathbf{D}^{-1}-\Psi^{D L}\right)^{-1} \mathbf{1}
$$

A similar discussion applies for the uplink. In that case, the power vector is denoted as $\mathbf{q}$. The SINR of a data stream $j$ of user $k$ in the uplink can be expressed as follows,

$$
\operatorname{SINR}_{k j}^{U L}=q_{k j} \frac{\text { um }_{k j}}{d e n_{k j}}
$$

where

$$
\operatorname{num}_{k j}=\left(\sum_{b=1}^{B} \mathbf{u}_{k j}^{(b)^{H}} \mathbf{H}_{k}^{(b)}\right) \mathbf{v}_{k j} \mathbf{v}_{k j}^{H}\left(\sum_{b=1}^{B} \mathbf{H}_{k}^{(b)^{H}} \mathbf{u}_{k j}^{(b)}\right)
$$

and

$$
\begin{aligned}
& \operatorname{den}_{k j}=\sum_{l=1 ; l \neq j}^{L_{k}}\left[q_{k l}\left|\left(\sum_{b=1}^{B} \mathbf{u}_{k j}^{(b)^{H}} \mathbf{H}_{k}^{(b)}\right) \mathbf{v}_{k l}\right|^{2}\right] \\
& +\mathbf{E}\left[\sum_{c=1 ; c \neq k}^{K} \sum_{l=1}^{L_{c}} q_{c l}\left|\sum_{b=1}^{B} \mathbf{u}_{k j}^{(b)^{H}} \mathbf{H}_{c}^{(b)} \mathbf{v}_{c l} \mathbf{e}_{c k j}^{(b)}\right|^{2}\right]+\sigma^{2} .
\end{aligned}
$$

Setting the same SINR targets as the downlink and grouping equalities of similar to (9), for the uplinl we get

$$
\mathbf{q} \frac{1}{C^{U L}}=\mathbf{D} \Psi^{U L} \mathbf{q}+\mathbf{D} \sigma^{U L}
$$

where

$$
\mathbf{D}=\operatorname{diag}\left(\frac{\Gamma_{11}}{\text { num }_{11}}, \frac{\Gamma_{12}}{\text { num }_{12}}, \ldots, \frac{\Gamma_{K L_{k}}}{\text { num }_{K L_{K}}}\right),
$$

$\left[\Psi^{U L}\right]_{L_{\text {row }} L_{\text {col }}}=\left|\mathbf{E}\left[\sum_{b=1}^{B} \mathbf{u}_{k_{\text {row }} l_{\text {row }}}^{(b)} \mathbf{H}_{k_{\text {col }}}^{(b)} \mathbf{v}_{k_{\text {col }} l_{\text {col }}} \mathbf{e}_{k_{\text {row }} k_{\text {col }} l_{\text {col }}}^{(b)}\right]\right|$

$$
1 \leq L_{\text {row }}, L_{\text {col }} \leq L \text { and } L_{\text {row }} \neq L_{\text {col }} \text {, }
$$

and

$$
\sigma^{U L}=\sigma^{2}\left[\sum_{b=1}^{B} \mathbf{u}_{11}^{H} \mathbf{u}_{11}, \ldots, \sum_{b=1}^{B} \mathbf{u}_{K L_{K}}^{H} \mathbf{u}_{K L_{K}}\right]^{T}=\sigma^{2} \mathbf{1} .
$$

Again, we have $\left[\Psi^{U L}\right]_{L_{\text {row }} L_{\text {col }}}=0$ when $L_{\text {row }}=L_{\text {col }}$, and $\mathbf{e}_{k k}^{(b)}=\mathbf{x}_{k}$. Note that the matrix $\mathbf{D}$ is equal in the downlink and uplink and hence it has no superscript. Similarly, by setting $C^{U L}=1$, we get

$$
\mathbf{q}=\sigma^{2}\left(\mathbf{D}^{-1}-\Psi^{U L}\right)^{-1} \mathbf{1}
$$

By establishing the above, we know that both the downlink and uplink have the same achievable SINR region, given no power constraints. To complete the first step, it is required that $\|\mathbf{p}\|_{1}$ equals $\|\mathbf{q}\|_{1}$. A sufficient condition for this equality to hold is to have $\mathbf{E}\left[\mathbf{i}_{j k}^{(b)} \mathbf{i}_{j k}^{(b)^{H}}\right]$ equal to $\mathbf{E}\left[\mathbf{e}_{j k}^{(b)} \mathbf{e}_{j k}^{(b)^{H}}\right]$, which makes $\Psi^{U L^{T}}$ equal to $\Psi^{D L}$, and hence $\|\mathbf{p}\|_{1}=\|\mathbf{q}\|_{1}$.

Unfortunately, for asynchronous interference, this condition does not necessarily hold since $\tau_{j k}^{(b)^{D L}}$ is not necessarily equal to $\tau_{j k}^{(b)^{U L}}$ for all values of $j, k$, and $b$. Moreover, setting up a system of equations $\left.\tau_{j k}^{(b)^{D L}}=\tau_{j k}^{(b)}\right)^{U L}$ and solving for virtual uplink delays can yield an over-determined system and no solution is guaranteed. Trying to solve for the virtual uplink delays using $\|\mathbf{p}\|_{1}=\|\mathbf{q}\|_{1}$ yields an under-determined system. Solutions can exist, but these solutions imply that users in the virtual uplink have to be redistributed for the actual propagation delays to match the solution obtained, which, in general, leads to contradictions. Therefore, whether any of these solutions are physically feasible is a question that remains to be examined in future work. What can be stated with confidence is that the simple, and extremely useful, form of the downlink-uplink duality does not exist in the case of asynchronous interference.

\section{A. Special Case: Synchronous Interference}

In a special case where synchronous interference is assumed, all propagation delays in the system are integer multiples of the symbol period $T_{s}$, considerable simplification occurs in the system model and all the analytical work that follows. The interference vectors $\mathbf{i}_{j k}^{(b)}$ and $\mathbf{e}_{j k}^{(b)}$ simplify to the data vector $\mathbf{x}_{j}$ of user $j$ and become independent of which BS transmits them. This happens because $\tau_{j k}^{(b)}$ (both in the downlink and uplink) becomes a multiple of $T_{s}$ and hence $\delta_{j k}^{(b)}=\tau_{j k}^{(b)} \bmod T_{S}=0, \rho\left(\delta_{j k}^{(b)}-T_{S}\right)=\rho\left(-T_{S}\right)=0$, and $\rho\left(\delta_{j k}^{(b)}\right)=\rho(0)=1$. Consequently, we get $\mathbf{E}\left[\mathbf{i}_{j k}^{(b)} \mathbf{i}_{j k}^{(b)^{H}}\right]=$ $\mathbf{E}\left[\mathbf{e}_{j k}^{(b)} \mathbf{e}_{j k}^{(b)^{H}}\right]=\mathbf{I}_{L_{k}}$, which is sufficient to complete the first step in proving downlink/uplink duality. Note that in this case, the system model can be viewed as the global system model in [4], except that the BS has $B \times M$ antennas. Moreover, the duality proof and a practical algorithm to achieve the required result are also the same as presented in [4]. Note that this is the approach taken in [7] which neglects the asynchronous nature of the inter-cell interference, an issue that has only recently been investigated [11]. The work in the available literature in this area is therefore, unfortunately, quite optimistic.

\section{B. Convexity of Power Allocation}

As we saw previously, the presence of asynchronous interference, at the very least, complicates the process of setting up a downlink/uplink duality. Even if this duality exists, we could proceed as before [2], [4] with developing an expression for the sum mean square error (SMSE) of all data streams and attempt to minimize it. In the uplink, the MSE error matrix 
for user $k$ can be expressed as follows.

$$
\begin{aligned}
& \mathbf{E}_{k}^{U L}=\mathbf{E}\left[(\hat{\mathbf{x}}-\mathbf{x})(\hat{\mathbf{x}}-\mathbf{x})^{H}\right] \\
& =\mathbf{U}_{k}^{H} \mathbf{H}_{k} \mathbf{V}_{k} \mathbf{Q}_{k} \mathbf{V}_{k}^{H} \mathbf{H}_{k}^{H} \mathbf{U}_{k}+\mathbf{U}_{k}^{H} \sum_{\substack{c=1 \\
c \neq k}}^{K} \mathbf{A}_{c k} \mathbf{U}_{k}+\sigma^{2} \mathbf{U}_{k}^{H} \mathbf{U}_{k} \\
& -\sqrt{\mathbf{Q}_{k}} \mathbf{V}_{k}^{H} \mathbf{H}_{k}^{H} \mathbf{U}_{k}-\mathbf{U}_{k}^{H} \mathbf{H}_{k} \mathbf{V}_{k} \sqrt{\mathbf{Q}_{k}}+\mathbf{I}_{L_{k}}
\end{aligned}
$$

where

$$
\begin{gathered}
\mathbf{U}_{k}=\left[\mathbf{U}_{k}^{(1)^{T}} \ldots \mathbf{U}_{k}^{(B)^{T}}\right]^{T}, \quad \mathbf{A}_{c k}=\left[\mathbf{A}_{c k}^{(1)^{T}} \ldots \mathbf{A}_{c k}^{(B)^{T}}\right]^{T} \\
\mathbf{A}_{c k}^{(b)^{T}}=\mathbf{H}_{c}^{(b)} \mathbf{V}_{c} \mathbf{Q}_{c} \mathbf{V}_{c}^{H} \mathbf{H}_{c}^{H} \boldsymbol{\Gamma}_{c k}^{(b)} \\
\boldsymbol{\Gamma}_{c k}^{(b)}=\operatorname{diag}\left[\gamma_{c k}^{(b, 1)} \mathbf{I}_{M}, \ldots, \gamma_{c k}^{(b, B)} \mathbf{I}_{M}\right]
\end{gathered}
$$

and

$$
\gamma_{c k}^{\left(b_{1}, b_{2}\right)} \mathbf{I}_{L_{c}}=\mathbf{E}\left[\mathbf{e}_{c k}^{\left(b_{1}\right)} \mathbf{e}_{c k}^{\left(b_{2}\right)^{H}}\right]
$$

Keeping all other matrices constant, the optimal solution for matrix $\mathbf{U}_{k}$ is the Weiner solution:

$\mathbf{U}_{k}=\left(\mathbf{H}_{k} \mathbf{V}_{k} \mathbf{Q}_{k} \mathbf{V}_{k}^{H} \mathbf{H}_{k}^{H}+\sum_{\substack{c=1 \\ c \neq k}}^{K} \mathbf{A}_{c k}+\sigma^{2} \mathbf{I}_{N_{k}}\right)^{-1} \mathbf{H}_{k} \mathbf{V}_{k} \sqrt{\mathbf{Q}_{k}}$

Substituting $\mathbf{U}_{k}$ back into (20), we get the following expression for $\mathbf{E}_{k}^{U L, M M S E}$.

$$
\mathbf{E}_{k}^{U L, M M S E}=\mathbf{I}_{L_{k}}-\sqrt{\mathbf{Q}_{k}} \mathbf{V}_{k}^{H} \mathbf{H}_{k}^{H} \mathbf{J}_{k}^{-1} \mathbf{H}_{k} \mathbf{V}_{k} \sqrt{\mathbf{Q}_{k}}
$$

where

$$
\mathbf{J}_{k}=\left(\mathbf{H}_{k} \mathbf{V}_{k} \mathbf{Q}_{k} \mathbf{V}_{k}^{H} \mathbf{H}_{k}^{H}+\sum_{j=1 ; j \neq k}^{K} \mathbf{A}_{j k}+\sigma^{2} \mathbf{I}\right) .
$$

Note that the matrix $\mathbf{J}_{k}$ cannot be made independent of user $k$ since $\mathbf{A}_{j k}$ is not common to all users. With the expression of the MSE of user $k$ available, the SMSE can be expressed as follows.

$$
\begin{aligned}
S M S E & =\sum_{k=1}^{K} \operatorname{tr}\left(\mathbf{E}_{k}^{U L, M M S E}\right) \\
& =\sum_{k=1}^{K} L_{k}-\sum_{k=1}^{K} \operatorname{tr}\left(\sqrt{\mathbf{Q}_{k}} \mathbf{V}_{k}^{H} \mathbf{H}_{k}^{H} \mathbf{J}_{k}^{-1} \mathbf{H}_{k} \mathbf{V}_{k} \sqrt{\mathbf{Q}_{k}}\right) \\
& =L-\sum_{k=1}^{K} \operatorname{tr}\left(\mathbf{H}_{k} \mathbf{V}_{k} \mathbf{Q}_{k} \mathbf{V}_{k}^{H} \mathbf{H}_{k}^{H} \mathbf{J}_{k}^{-1}\right)
\end{aligned}
$$

Given the complexity of determining the matrices $\mathbf{V}_{k}$ and $\mathbf{Q}_{k}$ for $k=1, \ldots, K$, that minimize the SMSE, a suboptimal, yet practical method involves iterating between fixing all $\mathbf{V}_{k}$ and solving for the matrices $\mathbf{Q}_{k}$ that minimize the SMSE, and fixing all $\mathbf{Q}_{k}$ and solving for the matrices $\mathbf{V}_{k}$ that minimize the SMSE. In the case of synchronous interference mentioned previously, this was facilitated by the fact that the SMSE expression proves to be convex in $\mathbf{Q}=\operatorname{diag}\left(\mathbf{Q}_{1}, \ldots, \mathbf{Q}_{K}\right)$. In the case of asynchronous interference, the dependence of
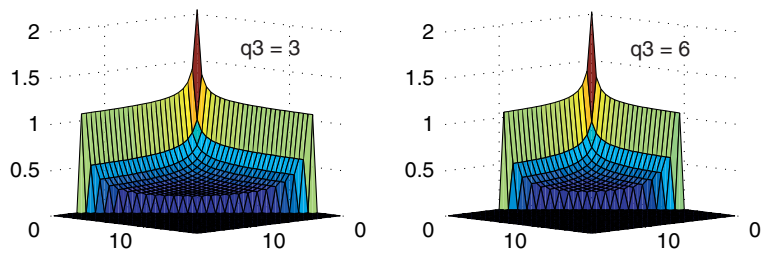

Fig. 1. SMSE versus powers of 3 users $(q 1, q 2, q 3)$ each with one data stream in asynchronous case. Power in Watts assuming $\sigma^{2}=1$ Watt.

the term $\mathbf{J}_{k}$ on the index $k$ complicates matters and proving convexity becomes difficult. However, our simulations suggest that convexity still holds (see figure 1).

If duality holds and the power allocation problem is convex, the algorithm to minimize SMSE while using multiple BSs is similar to that in [4]. The algorithm iterates between the matrices $\mathbf{U}, \mathbf{P}, \mathbf{V}$ and $\mathbf{Q}$, finding the optimal value for one while keeping the other three constant. The iterations terminate when a specified tolerance on the change in SMSE is reached.

\section{Simulation Results}

In this section, the simulation results for the synchronous and asynchronous interference cases are presented. Unless specified otherwise, the values of the variables used are:

$$
\begin{gathered}
B=2, K=2, M=4, N=\left[\begin{array}{ll}
2 & 2
\end{array}\right], L=\left[\begin{array}{ll}
2 & 2
\end{array}\right] \\
\sigma_{\text {AWGN }}^{2}=1, \sigma_{\text {Rayleigh }}^{2}=1, \operatorname{SNR}=\left(2 P_{\max } / \sigma_{A W G N}^{2}\right)
\end{gathered}
$$

\section{A. Synchronous Interference}

The performance, in terms of bit error rate (BER), of using the iterative SMSE minimization algorithm of [4] for the synchronous interference case is shown in figure 2. As can be seen, when all delays are integer multiples of $T_{s}$, the algorithm performs as well as expected.

\section{B. Asynchronous Interference}

In this subsection, three different approaches to the asynchronous case are simulated.

1) Assuming the Existence of Duality: When duality is assumed to exist, a variant of the iterative algorithm presented in [4] can be used, but with asynchronous data. This causes the derived downlink powers for users in a BS to sum up to a value less than or greater than $P_{\max }$, which is not optimal or not possible, respectively. Hence, the allocated powers are scaled with the same factor, such that their sum is $P_{\max }$. The BER performance of this approach, shown in figure 2, compares to the synchronous interference case at low SNRs. At high SNRs, the performance worsens, with the non-optimal linear scaling of power being a possible cause.

2) Nonlinear Programming: Nonlinear programming can be considered to be a "brute force" approach to solve the optimization problem of minimizing the SMSE. There is no guarantee of global convergence, however, the approach 


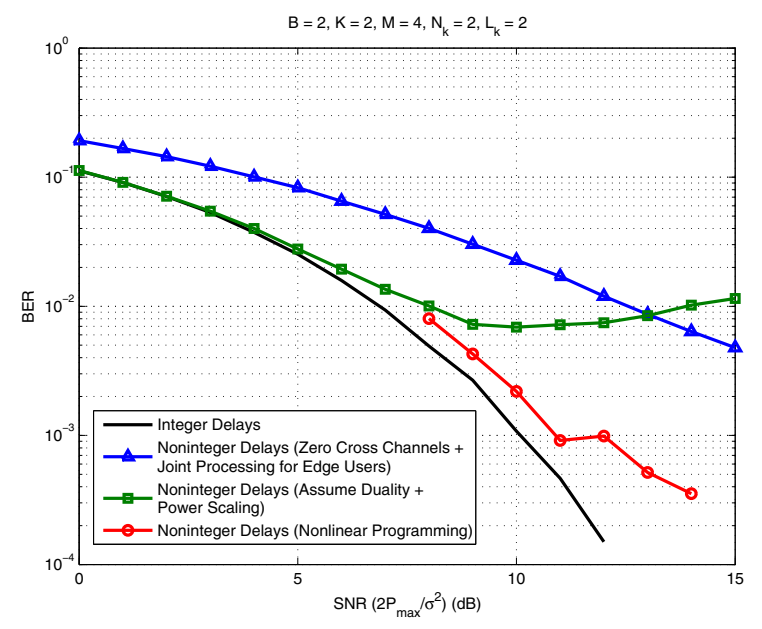

Fig. 2. BER plots for all simulations

does provide local solutions. In the asynchronous case, the optimization problem is:

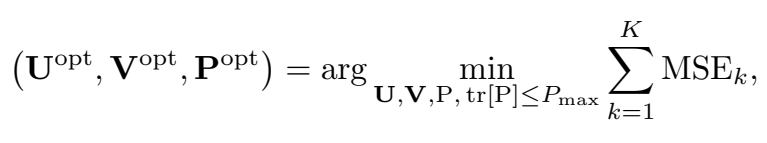

where $\operatorname{tr}[\cdot]$ represents the trace of a matrix. This approach creates a significantly higher computation load than iterative algorithms [1], [4]. However, its performance is close to the synchronous interference case as can be seen in figure 2 . This result is interesting in that it shows that cooperation via multiple base stations is possible despite the asynchronous interference. This motivates the search for a simpler, possibly iterative, algorithm that achieves similar performance with a lower computation load. Note, however, that there is still a huge performance loss with respect to the synchronous case.

3) Zero Power Cross Channels: When a user is close enough to a BS, it can be assumed that this user has a good channel to that $\mathrm{BS}$, and receives minimal interference from users in other cells. Hence, the cross channels corresponding to this user can be assumed to have zero power. On the other hand, edge users, which are at fairly similar distances to two BSs, can be assumed to have similar channels to those two BSs. Accordingly, it can be assumed that BSs transmit to all users at the same time, which eliminates the problem of asynchronous interference for non-edge users. As for edge users, it can be assumed that they use OFDM and utilize the cyclic prefix to mitigate asynchronous reception [12][15]. This changes the structure of some of the matrices used; however, the derivations remain similar to those presented in Section III, duality holds, and simulations suggest that the power allocation problem is convex (similar results to figure 1). Figure 2 shows the performance of this approach. At high SNRs, this approach provides better performance than the previous approach.

\section{CONCLUding REMARKS}

In this paper we investigated linear precoding for the multiuser MIMO case with multiple cooperating BSs. We showed that the downlink/uplink duality is no longer guaranteed due to the asynchronous interference introduced by the use of multiple BSs. While the rearrangement of users in the virtual uplink may reestablish this duality, the resulting propagation delays might be contradictory. Even if duality was assumed to exist, the asynchronous nature of the interference poses difficulties in the further analysis, such as the convexity of the power allocation problem, which is a basic step required in trying to minimize the SMSE. Moreover, the asynchronous interference proves detrimental to the BER performance of the iterative algorithm, with the duality assumption still in place. Moreover, even when the cross channels are assumed to have zero power, the performance is still far from what can be potentially achieved, as demonstrated by the nonlinear programming simulation. However, the zero power cross channels case motivates the use of OFDM and its numerous benefits to battle asynchronism. This leaves the design of effective algorithms for the truly asynchronous case an open problem.

\section{REFERENCES}

[1] A. J. Tenenbaum and R. S. Adve, "Joint multiuser transmit-receive optimization using linear processing," in Proc. IEEE ICC, vol. 1, Paris, France, June 2004, pp. 588-592.

[2] M. Schubert and H. Boche, "Solution of the multiuser downlink beamforming problem with individual SINR constraints," IEEE Trans. Veh. Technol., vol. 53, no. 1, pp. 18-28, Jan. 2004.

[3] S. Shi and M. Schubert, "MMSE transmit optimization for multi-user multi-antenna systems," in Proc. IEEE ICASSP 05, Mar. 2005.

[4] A. M. Khachan, A. J. Tenenbaum, and R. S. Adve, "Linear processing for the downlink in multiuser MIMO systems with multiple data streams," in Proc. IEEE ICC, June 2006.

[5] M. Codreanu, A. Tölli, M. Juntti, and M. Latva-aho, "Joint design of TxRx beamformers in MIMO downlink channel," IEEE Trans. on Signal Proc., vol. 55, no. 9, pp. 4639-4655, September 2007.

[6] E. Visotsky and U. Madhow, "Optimum beamforming using transmit antenna arrays," in Proc. IEEE VTC Spring, May 1999, pp. 851-856.

[7] A. Tolli, M. Codreanu, and M. Juntti, "Linear cooperative multiuser MIMO transciever design with per BS power constraints," in Proc. IEEE ICC 07, Glasgow, Scotland, June 2007.

[8] H. Dai, A. F. Molisch, , and H. V. Poor, "Downlink capacity of interference-limited MIMO systems with joint detection," IEEE Trans. on Wireless Commun., vol. 3, pp. 442-453, March 2004.

[9] S. Jafar, G. Foschini, and A. Goldsmith, "Phantomnet: Exploring optimal multicellular multiple antenna systems," EURASIP J. App. Sig. Proc., pp. 591-604, October 2004.

[10] B. L. Ng, J. S. Evans, S. V. Hanly, and D. Aktas, "Transmit beamforming with cooperative base stations," in Proc. IEEE ISIT, 2005, pp. 14311435 .

[11] H. Zhang, N. B. Mehta, A. F. Molisch, J. Zhang, and H. Dai, "On the fundamentally asynchronous nature of interference in cooperative base station systems," in Proc. IEEE ICC, Glasgow, Scotland, June 2007.

[12] T. A. Thomas and F. W. Vook, "Asynchronous interference suppression in broadband cyclic-prefix communications," in Proc. IEEE WCNC, vol. 1, Mar. 2003, pp. 568-572.

[13] H. Cheon and D. Hong, "A blind spatio-temporal equalizer using cyclic prefix in OFDM systems," in IEEE ICASSP, vol. 5, June 2000, pp. 2957-2960.

[14] S. Prakriya, "Equalization and block-synchronization for OFDM signals," in IEEE ICASSP, vol. 4, May 2001, pp. 2317-2320.

[15] A. Bletsas and A. Lippman, "Efficient collaborative (viral) communication in OFDM based wlans," in Intl. Symp. on Adv. Radio Tech. (ISART 2003), Boulder, Colorado, Mar. 2003. 